\title{
NORM INEQUALITIES OF DAVIDSON-POWER TYPE
}

\section{Ahmad Al-Natoor, Wasim Audeh And Fuad Kittaneh}

Abstract. Let $A, B$, and $X$ be $n \times n$ complex matrices such that $A$ and $B$ are positive semidefinite. It is shown, among other inequalities, that

$$
\|A X+X B\| \leqslant \frac{1}{2} \max \left(\|A\|,\left\|X B X^{*}\right\|\right)+\frac{1}{2} \max \left(\left\|X^{*} A X\right\|,\|B\|\right)+\left\|A^{1 / 2} X B^{1 / 2}\right\| .
$$

This norm inequality extends an inequality of Kittaneh, which improves an earlier inequality of Davidson and Power.

Mathematics subject classification (2010): 15A60, 15A18, 15A42, 47A30, 47B15.

Keywords and phrases: Concave function, positive semidefinite matrix, singular value, unitarily invariant norm, inequality.

\section{REFERENCES}

[1] R. Bhatia, Matrix Analysis, Springer, New York, 1997.

[2] R. Bhatia AND F. KitTAneH, On the singular values of a product of operators, SIAM J. Matrix Anal. Appl. 11 (1990), 272-277.

[3] J.-C. Bourin, Convexity or concavity inequalities for Hermitian operators, Math. Ineq. Appl. 7 (2004), 607-620.

[4] J.-C. Bourin, A matrix subadditivity inequality for symmetric norms, Proc. Amer. Math. Soc. 138 (2009), 495-504.

[5] J.-C. Bourin and A. Mhanna, Positive block matrices and numerical ranges, C.R. Acad. Sci. Paris 355(2017), 1077-1081.

[6] K. Davidson, S. C. Power, Best approximation in $C^{*}$-algebras, J. Reine Angew. Math. 368 (1986), 43-62.

[7] R. A. Horn And C. R. Johnson, Matrix Analysis, 2nd Edition, Cambridge University Press, 2013.

[8] J. C. Hou AND H. K. DU, Norm inequalities of positive operator matrices, Integral Equations Operator Theory 22(1995), 281-294.

[9] F. Kittaneh, Norm inequalities for certain operator sums, J. Funct. Anal. 143 (1997), 337-348.

[10] F. Kittaneh, Norm inequalities for sums of positive operators, J. Operator Theory 48 (2002), 95103.

[11] F. Kittaneh, Inequalities for commutators of positive operators, J. Funct. Anal. 250 (2007), 132143.

[12] X. ZHAN, Singular values of differences of positive semidefinite matrices, SIAM J. Matrix Anal. Appl. 22 (2002), 819-823. 